\title{
NLO forward jet vertex
}

\author{
F. Caporale*, D.Yu. Ivanov ${ }^{\dagger}$, B. Murdaca*, A. Papa* and A. Perri* \\ *Dipartimento di Fisica, Università della Calabria, and Istituto Nazionale di Fisica Nucleare, \\ Gruppo collegato di Cosenza, I-87036 Arcavacata di Rende, Cosenza, Italy \\ ${ }^{\dagger}$ Sobolev Institute of Mathematics and Novosibirsk State University, 630090 Novosibirsk, Russia
}

\begin{abstract}
We calculate in the BFKL approach the jet vertex relevant for the production of MuellerNavelet jets in proton-proton collisions. We consider both cases of incoming quark and gluon and show explicitly that all infrared divergences cancel when renormalized parton densities are considered. Finally we compare our expression for the vertex with a previous calculation [1].
\end{abstract}

Keywords: perturbative QCD, BFKL approach, jets, LHC

PACS: $12.38 .-\mathrm{t}, 12.38 . \mathrm{Cy}$

\section{INTRODUCTION}

The large center-of-mass energy of hadron colliders like the Tevatron and the Large Hadron Collider (LHC) is not only useful for the production of possible new heavy particles, but also allows to investigate the high-energy regime of QCD. An especially interesting situation is the production of Mueller-Navelet jets [2]. This inclusive process $p\left(p_{1}\right)+p\left(p_{2}\right) \rightarrow J_{1}\left(k_{J_{1}}\right)+J_{2}\left(k_{J_{2}}\right)+X$ was proposed to study the Regge limit of perturbative QCD in proton-proton collision.

Since the two jets produced have transverse momenta much larger than the QCD scale, $\vec{k}_{J_{1}}^{2} \sim \vec{k}_{J_{2}}^{2} \gg \Lambda_{\mathrm{QCD}}^{2}$, it is possible to use perturbative QCD. Moreover, they are separated by a large interval of rapidity, $\Delta y \gg 1$, which means large center of mass energy $\sqrt{s}$ of the proton collisions, $s=2 p_{1} \cdot p_{2} \gg \vec{k}_{J 1,2}^{2}$. Since large logarithms of the energy compensate the small QCD coupling, they must be resummed to all orders of perturbation theory. The BFKL approach [3] is the most suitable framework for the theoretical description of the high-energy limit of hard or semi-hard processes. It provides indeed a systematic way to perform the resummation of the energy logarithms, both in the leading logarithmic approximation (LLA), which means resummation of all terms $\left(\alpha_{s} \ln (s)\right)^{n}$, and in the next-to-leading logarithmic approximation (NLA), which means resummation of all terms $\alpha_{s}\left(\alpha_{s} \ln (s)\right)^{n}$. In QCD collinear factorization the cross section of the process reads

$$
\frac{d \sigma}{d J_{1} d J_{2}}=\sum_{i, j=q, \bar{q}, g} \int_{0}^{1} \int_{0}^{1} d x_{1} d x_{2} f_{i}\left(x_{1}, \mu_{F}\right) f_{j}\left(x_{2}, \mu_{F}\right) \frac{d \hat{\sigma}_{i, j}\left(x_{1} x_{2} s, \mu_{F}\right)}{d J_{1} d J_{2}},
$$

with $d J_{1,2}=d x_{J_{1,2}} d^{D-2} k_{J_{1,2}}$ and the $i, j$ indices specify parton types (quarks $q$, antiquarks $\bar{q}$ or gluon $g) ; f_{i}\left(x, \mu_{F}\right)$ denotes the initial proton parton density function (PDF), the longitudinal fractions of the partons involved in the hard subprocess are $x_{1,2}$, while $\mu_{F}$ is the factorization scale and $d \hat{\sigma}_{i, j}\left(x_{1} x_{2} s, \mu_{F}\right)$ is the partonic cross section for the 
production of jets, $\hat{s}=x_{1} x_{2} s$ being the energy of the parton-parton collision. In the BFKL approach the resummed cross section of the hard subprocess reads

$$
\begin{aligned}
\frac{d \hat{\sigma}}{d J_{1} d J_{2}}=\frac{1}{(2 \pi)^{D-2}} & \int \frac{d^{D-2} q_{1}}{\vec{q}_{1}^{2}} \frac{d \Phi_{J_{1}}\left(\vec{q}_{1}, s_{0}\right)}{d J_{1}} \int \frac{d^{D-2} q_{2}}{\vec{q}_{2}^{2}} \frac{d \Phi_{J_{2}}\left(-\vec{q}_{2}, s_{0}\right)}{d J_{2}} \\
& \times \int_{\delta-i \infty}^{\delta+i \infty} \frac{d \omega}{2 \pi i}\left(\frac{\hat{s}}{s_{0}}\right)^{\omega} G_{\omega}\left(\vec{q}_{1}, \vec{q}_{2}\right),
\end{aligned}
$$

that is a convolution of the jet impact factors $d \Phi_{i} / d J_{i}$ with the Green's function $G_{\omega}$, process-independent and determined through the BFKL equation.

The aim of this work is the recalculation of next-to-leading order (NLO) jet vertex, first found by Bartels et al. [1], in order to have an independent check of their results.

The starting point of the calculation are the impact factors for colliding partons [4, 5, 6, 7], which describe the totally inclusive transition of a parton into a group of partons (one or two in the NLO). In order to select the parton(s) in the final state that will generate the jet, we "open" one of the integrations over the partonic phase space: for the one-parton case, we introduce a function $S_{J}^{(2)}$ that identifies the jet momentum with that of the parton; for the two-parton state, we introduce a function $S_{J}^{(3)}$ that identifies the jet momentum with the momentum of one of the two partons or with the sum of the momenta of two partons. In the calculation of the jet vertex, the infrared divergences related with soft emission will cancel in the sum with virtual corrections. The remaining infrared divergences are taken care of by the PDFs' renormalization. The collinear counterterms appear due to the replacement of the bare PDFs by the renormalized physical quantities obeying DGLAP evolution equations. Ultraviolet divergences are removed by the counterterm related with QCD charge renormalization. The LO jet impact reads:

$$
\frac{d \Phi_{J}^{(0)}(\vec{q})}{d J}=\Phi_{q}^{(0)} \int_{0}^{1} d x\left(\frac{C_{A}}{C_{F}} f_{g}(x)+\sum_{a=q, \bar{q}} f_{a}(x)\right) S_{J}^{(2)}(\vec{q} ; x),
$$

where $\Phi_{q}^{(0)}=g^{2} \frac{\sqrt{N_{c}^{2}-1}}{2 N_{c}}$ is the quark impact factor at the Born level, $\vec{q}$ is the Reggeon momentum, $S_{J}^{(2)}$ is the selection function previously defined, and $f_{g}$ and $f_{a}$ the gluon and quark PDFs, respectively. Substituting in Eq. (1) the bare QCD coupling and bare PDFs by the renormalized ones (in the $\overline{\mathrm{MS}}$ scheme), we obtain the following expressions

$$
\begin{gathered}
\frac{\left.d \Phi_{J}(\vec{q})\right|_{\text {charge c.t. }}}{d J}=\frac{\alpha_{s}}{2 \pi}\left(\frac{1}{\hat{\varepsilon}}+\ln \frac{\mu_{R}^{2}}{\mu^{2}}\right)\left(\frac{11 C_{A}}{6}-\frac{N_{F}}{3}\right) \Phi_{q}^{(0)} \\
\times \int_{0}^{1} d x\left(\frac{C_{A}}{C_{F}} f_{g}(x)+\sum_{a=q, \bar{q}} f_{a}(x)\right) S_{J}^{(2)}(\vec{q} ; x) \\
\frac{\left.d \Phi_{J}(\vec{q})\right|_{\text {collinear c.t. }}}{d J}=-\frac{\alpha_{s}}{2 \pi}\left(\frac{1}{\hat{\varepsilon}}+\ln \frac{\mu_{F}^{2}}{\mu^{2}}\right) \Phi_{q}^{(0)} \int_{0}^{1} d \beta \int_{0}^{1} d x S_{J}^{(2)}(\vec{q} ; \beta x)
\end{gathered}
$$




$$
\times\left[\sum_{a=q, \bar{q}}\left(P_{q q}(\beta) f_{a}(x)+P_{q g}(\beta) f_{g}(x)\right)+\frac{C_{A}}{C_{F}}\left(P_{g g}(\beta) f_{g}(x)+P_{g q}(\beta) \sum_{a=q, \bar{q}} f_{a}(x)\right)\right],
$$

for the charge renormalization and the collinear counterterms, respectively (here $\mu$ is the scale introduced by the dimensional regularization).

Now we have all the necessary ingredients to perform our calculation of the NLO corrections to the jet impact factor. We will consider separately the subprocesses initiated by the quark and the gluon PDFs and denote

$$
V=V_{q}+V_{g} \quad \text { with } \quad \frac{d \Phi_{J}^{(1)}(\vec{q})}{d J} \equiv \frac{\alpha_{s}}{2 \pi} \Phi_{q}^{(0)} V(\vec{q})
$$

\section{NLO JET IMPACT FACTOR}

In the quark case, virtual corrections are the same as in the case of the inclusive quark impact factor [4, 5, 6]:

$$
\begin{aligned}
& V_{q}^{(V)}(\vec{q})=-\frac{\Gamma[1-\varepsilon]}{\varepsilon(4 \pi)^{\varepsilon}} \frac{\Gamma^{2}(1+\varepsilon)}{\Gamma(1+2 \varepsilon)} \int_{0}^{1} d x \sum_{a=q, \bar{q}} f_{a}(x) S_{J}^{(2)}(\vec{q} ; x) \\
& \times\left[C_{F}\left(\frac{2}{\varepsilon}-3\right)-\frac{N_{F}}{3}+C_{A}\left(\ln \frac{s_{0}}{\vec{q}^{2}}+\frac{11}{6}\right)\right]+\text { finite terms } .
\end{aligned}
$$

For the incoming quark case, real corrections originate from the quark-gluon production process. If we denote the transverse momentum of the gluon by $k$ and its longitudinal fraction by $\beta x$, then the real contribution has the form

$$
\begin{aligned}
& V_{q}^{(R)}(\vec{q})=\int_{0}^{1} d x \sum_{a=q, \bar{q}} f_{a}(x)\left\{\frac { \Gamma [ 1 - \varepsilon ] } { \varepsilon ( 4 \pi ) ^ { \varepsilon } } \frac { \Gamma ^ { 2 } ( 1 + \varepsilon ) } { \Gamma ( 1 + 2 \varepsilon ) } \left[C_{F}\left(\frac{2}{\varepsilon}-3\right) S_{J}^{(2)}(\vec{q} ; x)\right.\right. \\
& \left.\quad+\int_{0}^{1} d \beta\left(P_{q q}(\beta)+\frac{C_{A}}{C_{F}} P_{g q}(\beta)\right) S_{J}^{(2)}(\vec{q} ; x \beta)\right]+\frac{C_{A}}{(4 \pi)^{\varepsilon}} \int \frac{d^{D-2} k}{\pi^{1+\varepsilon}} \\
& \left.\quad \times \frac{\vec{q}^{2}}{\vec{k}^{2}(\vec{q}-\vec{k})^{2}} \ln \frac{s_{0}}{(|\vec{k}|+|\vec{q}-\vec{k}|)^{2}} S_{J}^{(2)}(\vec{q}-\vec{k} ; x)\right\}+ \text { finite terms }
\end{aligned}
$$

Also in the gluon case the virtual corrections are the same as for the inclusive gluon impact factor [4, 6]:

$$
\begin{aligned}
& V_{g}^{(V)}(\vec{q})=-\frac{\Gamma[1-\varepsilon]}{\varepsilon(4 \pi)^{\varepsilon}} \frac{\Gamma^{2}(1+\varepsilon)}{\Gamma(1+2 \varepsilon)} \int_{0}^{1} d x \frac{C_{A}}{C_{F}} f_{g}(x) S_{J}^{(2)}(\vec{q} ; x) \\
& \times\left[C_{A} \ln \left(\frac{s_{0}}{\vec{q}^{2}}\right)+C_{A}\left(\frac{2}{\varepsilon}-\frac{11}{6}\right)+\frac{N_{F}}{3}\right]+\text { finite terms } .
\end{aligned}
$$


In the NLO gluon impact factor real corrections come from the production of quarkantiquark state and a gluon-gluon state [4, 6, 7]. We find

$$
\begin{gathered}
V_{g}^{(R)}(\vec{q})=\frac{\Gamma[1-\varepsilon]}{\varepsilon(4 \pi)^{\varepsilon}} \frac{\Gamma^{2}(1+\varepsilon)}{\Gamma(1+2 \varepsilon)} \int_{0}^{1} d x f_{g}(x)\left\{\frac{C_{A}}{C_{F}}\left(\frac{N_{F}}{3}+\frac{2 C_{A}}{\varepsilon}-\frac{11}{6} C_{A}\right)\right. \\
\times S_{J}^{(2)}(\vec{q} ; x)+\int_{0}^{1} d \beta\left[2 N_{F} P_{q g}(\beta)+2 C_{A} \frac{C_{A}}{C_{F}}\left(P(\beta)+\frac{(1-\beta) P(1-\beta)}{(1-\beta)_{+}}\right)\right] \\
\left.\times S_{J}^{(2)}(\vec{q} ; x \beta)\right\}+\frac{C_{A}}{(4 \pi)^{\varepsilon}} \int_{0}^{1} d x \frac{C_{A}}{C_{F}} f_{g}(x) \int \frac{d^{D-2} k}{\pi^{1+\varepsilon}} \frac{\vec{q}^{2}}{\vec{k}^{2}(\vec{k}-\vec{q})^{2}} \ln \frac{s_{0}}{(|\vec{k}|+|\vec{q}-\vec{k}|)^{2}} \\
\times S_{J}^{(2)}(\vec{q}-\vec{k} ; x)+\text { finite terms . }
\end{gathered}
$$

To conclude, we collect the contributions given in Eqs. (2), (3), (5)-(8), and we note that we are left with two divergences: the last terms of (6) and of (8). It easy to see that the convolution of the jet vertex with BFKL Green's function, required for the calculation of the jet cross section, will give a divergence-free result.

More details about this calculation can be found in Ref. [8].

\section{SUMMARY}

We have recalculated the jet production vertex, first found by Bartels et al. [1], in a more direct way, starting from the known general expression of NLO impact factors, given in Ref. [9], and applied to the case of parton impact factors. Nevertheless, in many technical steps we followed closely the derivation of Refs. [1]. In our approach the energy scale $s_{0}$ is not fixed. Performing the transition (see [10]) from the standard BFKL scheme with arbitrary energy scale $s_{0}$ to the one used in [1], where the energy scale depends on the Reggeon momentum, we can see a complete agreement with Refs. [1].

The jet vertex discussed in this paper is an essential ingredient also for the study of the forward jet production in deep inelastic scattering in the NLA.

\section{REFERENCES}

1. J. Bartels, D. Colferai, G. Vacca, Eur. Phys. J C24, 83 (2002); C29, 235 (2003).

2. A.H. Mueller, H. Navelet, Nucl. Phys. B282, 727 (1987).

3. V.S. Fadin, E.A. Kuraev, L.N. Lipatov, Phys. Lett. B60, 50 (1975); E.A. Kuraev, L.N. Lipatov and V.S. Fadin, Zh. Eksp. Teor. Fiz. 71, 840 (1976) [Sov. Phys. JETP 44, 443 (1976)]; 72, 377 (1977) [45, 199 (1977)]; Ya.Ya. Balitskii and L.N. Lipatov, Sov. J. Nucl. Phys. 28, 822 (1978).

4. V.S. Fadin, R. Fiore, M.I. Kotsky, A. Papa, Phys. Lett. D61, 094005 (2000).

5. V.S. Fadin, R. Fiore, M.I. Kotsky, A. Papa, Phys. Lett. D61, 094006 (2000)

6. M. Ciafaloni, D. Colferai, Nucl. Phys. B 538, 187 (1999).

7. M. Ciafaloni, Phys. Lett. B 429, 363 (1998).

8. F. Caporale, D. Yu. Ivanov, B. Murdaca, A. Papa, A. Perri, JHEP 1202, 101 (2012).

9. V.S. Fadin, et al., Phys. Lett. B 440, 359 (1998).

10. V.S. Fadin, arXiv:hep-ph/9807527. 